\title{
Evaluación positiva de medicamentos: junio, julio y agosto 2015
}

\author{
Prats Olivan P. ${ }^{\text {aa }}$, Sánchez López P. ${ }^{1 a}$, Aparicio Hernández R. ${ }^{2 b}$, García Luque A. ${ }^{3 b}$ \\ Sanid. mil. 2015; 71 (4): 252-256; ISSN: 1887-8571
}

\begin{abstract}
RESUMEN
Se reseñan los medicamentos evaluados y con dictamen positivo por la comisión de expertos de la Agencia Española de Medicamentos y Productos Sanitarios o de la Agencia Europea del Medicamento hechos públicos en junio y julio de 2015. Se trata de opiniones técnicas positivas previas a la autorización y puesta en el mercado del medicamento.

PALABRAS CLAVE: Alirocumab, Praluent ${ }^{\circledR}$, Asfotasa Alfa, Strensiq ${ }^{\circledR}$, Ceftolozano/Tazobactam Zerbaxa ${ }^{\circledR}$, Complejo de Coordinación Citrato Férrico, Fexeric ${ }^{\circledR}$, Guanfacina, Intuniv ${ }^{\circledR}$, Inhibidor Alfa 1-Proteinasa Humana, Respreeza ${ }^{\circledR}$, Isavuconazol, Cresemba ${ }^{\circledR}$, Panobinostat, Farydak $^{\circledR}$, Plasmodium Falciparum y Vacuna contra la Hepatitis B Recombinante Adyuvada, Mosquirix ${ }^{\circledR}$, Sebelipasa Alfa, Kanuma ${ }^{\circledR}$, Sonidegib, Odomzo ${ }^{\circledR}$, Susoctocog Alfa, Obizur ${ }^{\circledR}$.
\end{abstract}

\section{Positive assessment of drugs: June, July and August 2015}

SUMMARY: The drugs assessed by the Spanish Agency for Medicines and Health Products or European Medicines Agency made public in june and july of 2015, and considered of interest to the healthcare professional, are reviewed. These are positive technical reports prior to the authorization and placing on the market of the product.

KEY WORDS: Alirocumab, Praluent ${ }^{\circledR}$, Asfotasa Alfa, Strensiq ${ }^{\circledR}$, Ceftolozano/Tazobactam Zerbaxa ${ }^{\circledR}$, Complejo de Coordinación Citrato Férrico, Fexeric ${ }^{\circledR}$, Guanfacina, Intuniv ${ }^{\circledR}$, Inhibidor Alfa 1-Proteinasa Humana, Respreeza ${ }^{\circledR}$, Isavuconazol, Cresemba ${ }^{\circledR}$, Panobinostat, Farydak $^{\circledR}$, Plasmodium Falciparum y Vacuna contra la Hepatitis B Recombinante Adyuvada, Mosquirix ${ }^{\circledR}$, Sebelipasa Alfa, Kanuma ${ }^{\circledR}$, Sonidegib, Odomzo $^{\circledR}$, Susoctocog Alfa, Obizur ${ }^{\circledR}$.

\section{ALIROCUMAB (Praluent $\left.^{\mathbb{R}}\right)^{1-4}$}

El principio activo es alirocumab, un agente modificador de lípidos. Alirocumab es un anticuerpo monoclonal humano, que se une selectivamente a la proproteína convertasa subtilisina kexina tipo 9 (PCSK9), una proteína que regula el reciclaje de los receptores de las lipoproteínas de baja densidad (LDL) en la superficie de las células del hígado disminuyendo la capacidad del hígado de eliminar el LDL de la sangre. Mediante la unión a PCSK9, alirocumab aumenta los niveles de los receptores de LDL en la superficie de las células hepáticas reduciendo los niveles séricos de LDL-colesterol.

Está indicado en adultos con hipercolesterolemia primaria (familiar heterocigótica y no familiar) o dislipidemia mixta, como tratamiento coadyuvante a la dieta:

- En combinación con estatinas o con estatinas junto con otros medicamentos hipolipemiantes en pacientes incapaces de al-

\footnotetext{
${ }^{1}$ Cte. Farmacéutico.

${ }^{2}$ MIR.

${ }^{3}$ Cte. Médico.

a Servicio de Farmacia Hospitalaria.

b Servicio de Farmacología Clínica. Hospital Central de la Defensa Gómez Ulla. Madrid. España.
}

Dirección para correspondencia: Servicio de Farmacia Hospitalaria. Hospital Central de la Defensa Gómez Ulla. 28047 Madrid. España. ppraoli@ea.mde.es

Recibido: 2 de octubre de 2015

Aceptado: 9 de octubre de 2015 canzar niveles óptimos de LDL-C a dosis máximas toleradas de estatinas o,

- Sola o en combinación con otros medicamentos hipolipemiantes en pacientes en los que el tratamiento con estatinas está contraindicado o no es bien tolerado.

El efecto de Praluent ${ }^{\circledR}$ sobre la morbilidad y mortalidad cardiovascular no ha sido determinado.

En los ensayos clínicos ha mostrado que reduce los niveles séricos de LDL-colesterol en los pacientes que no son capaces de controlar los niveles de colesterol, a pesar de recibir las dosis máximas toleradas de estatinas o que no son aptos para un tratamiento con estatinas.

Su eficacia y seguridad ha sido evaluada en 5 ensayos controlados con placebo, con 2.476 participantes expuestos al principio activo. Todos los participantes tenían hipercolesterolemia familiar hereditaria o de lo contrario tenían riesgo elevado de tener un ataque cardíaco o derrame cerebral y estaban tomando dosis máximas toleradas de estatinas, con o sin otras terapias modificadoras de lípidos. Los participantes que tomaron Praluent ${ }^{\mathbb{R}}$ tuvieron una reducción promedio de colesterol LDL entre 36 y 59 por ciento en comparación con el placebo.

Las reacciones adversas más comunes observadas durante el desarrollo clínico fueron signos y síntomas del tracto respiratorio superior, prurito y reacciones en el lugar de la inyección. El uso de Praluent ${ }^{\circledR}$ puede dar lugar a niveles muy bajos de colesterol; su impacto en la seguridad a largo plazo no ha sido establecido aún. 


\section{ASFOTASA ALFA $\left(\text { Strensiq }^{\circledR}\right)^{5,6}$}

El principio activo es asfotasa alfa, una enzima de reemplazo para suplementar la actividad de la fosfatasa alcalina no específica de tejido. Se piensa que ejerce su acción promoviendo la mineralización del esqueleto en pacientes con hipofosfatasia de inicio infantil.

Está indicada para el tratamiento de sustitución enzimática a largo plazo en pacientes con hipofosfatasia de inicio infantil, para tratar las manifestaciones óseas de la enfermedad. En base a los datos de eficacia y seguridad aportados se recomienda una autorización bajo circunstancias excepcionales. La autorización en circunstancias excepcionales está sujeta a ciertas obligaciones específicas, y se revisa de forma anual. Esto sucede cuando el solicitante no es capaz de proporcionar datos exhaustivos sobre eficacia y seguridad del medicamento, debido a la baja frecuencia de la enfermedad para la que está destinada, conocimientos científicos limitados en el área concerniente o consideraciones éticas relacionadas con la recogida de datos.

Ha mostrado mejorar la estructura ósea, medido por los cambios radiológicos en articulaciones, por cambios histológicos en biopsias de tejido óseo y por un aumento de la velocidad de crecimiento en algunos pacientes.

Las reacciones adversas más comunes observadas durante el desarrollo clínico fueron reacciones en el lugar de la inyección y reacciones asociadas a la inyección, generalmente de intensidad leve a moderada.

Debe ser prescrito por médicos con experiencia en el tratamiento de pacientes con desórdenes metabólicos u óseos.

Strensiq ${ }^{\circledR}$ fue designado como medicamento huérfano el 3 de diciembre de 2008.

\section{CEFTOLOZANO/TAZOBACTAM $\left(\operatorname{Zerbaxa}^{\circledR}\right)^{1,7,8}$}

Los principios activos son ceftolozano/tazobactam, una cefalosporina y un inhibidor de beta-lactamasa respectivamente. Ceftolozano ejerce su actividad bactericida mediante la unión a proteínas relevantes en la unión a penicilina, dando lugar a la inhibición de la síntesis de la pared celular bacteriana y la muerte celular. Tazobactam no ejerce ninguna actividad antibacteriana pero protege a ceftolozano frente a la hidrólisis.

Está indicado en adultos para el tratamiento de las siguientes infecciones:

- Infecciones complicadas intraabdominales (cIAI)

- Pielonefritis aguda

- Infecciones complicadas del tracto urinario (cITU)

En un ensayo randomizado, doble ciego, controlado, de no inferioridad, en el que se incluyeron 1083 pacientes con cITU o pielonefritis, que fueron randomizados para recibir levofloxacino o ceftolozano-tazobactam, este último mostró su no inferioridad frente al levofloxacino.

Se deben tener en consideración las recomendaciones oficiales sobre el uso adecuado de agentes antibacterianos.

Las reacciones adversas más comunes observadas durante el desarrollo clínico fueron: náuseas, dolor de cabeza, estreñimiento, diarrea y fiebre. Estos efectos fueron generalmente leves o moderados.

\section{COMPLEJO DE COORDINACIÓN CITRATO FÉRRICO $\left(\text { Fexeric }^{\circledR}\right)^{1,9,10}$}

El principio activo es un complejo de coordinación de citrato férrico.

El componente de hierro reacciona con el fosfato de la dieta en el aparato gastrointestinal (GI) y precipita el fosfato en forma de fosfato férrico. Este compuesto es insoluble y se excreta en heces, reduciendo la cantidad de fosfato que se absorbe del aparato GI. Por la unión del fosfato en el aparato GI y la reducción de la absorción, Fexeric $^{\circledR}$ reduce los niveles séricos de fósforo. Tras la absorción, el citrato se convierte en bicarbonato por los tejidos.

Está indicado para el control de la hiperfosfatemia en pacientes adultos con enfermedad renal crónica.

Este fármaco ha mostrado ser efectivo en la reducción de los niveles de fosfato en sangre en dos ensayos clínicos. En el primero, realizado sobre 359 pacientes con enfermedad renal crónica, después de 12 semanas ambos tratamientos lograron reducir los niveles de fosfato en $2 \mathrm{mg} / \mathrm{dl}$. En el segundo estudio, realizado en 149 pacientes, frente a placebo, mostró una reducción media de los niveles de fosfato de $0,7 \mathrm{mg} / \mathrm{dl}$, frente a $0,3 \mathrm{mg} / \mathrm{dl} \mathrm{del}$ placebo.

Las reacciones adversas más comunes observadas durante el desarrollo clínico fueron trastornos gastrointestinales.

\section{GUANFACINA $\left(\text { Intuniv }^{\circledR}\right)^{1,11}$}

El principio activo es guanfacina, un antihipertensivo conocido. Es un agonista selectivo del receptor adrenérgico alfa-2A. No se ha establecido totalmente el modo de acción de guanfacina en el Trastorno por Déficit de Atención e Hiperactividad (TDAH). Las investigaciones preclínicas sugieren que guanfacina modula la señalización en la corteza prefrontal y los ganglios basales mediante la modificación directa de la transmisión sináptica de noradrenalina en los receptores adrenérgicos alfa 2.

Está indicado en el tratamiento del TDAH en niños y adolescentes de 6 a 17 años para quienes los estimulantes no son adecuados, no se toleran o han demostrado ser ineficaces. Intuniv ${ }^{\circledR}$ debe ser administrado como parte de un programa de tratamiento integral del TDAH, que incluya medidas psicológicas, educativas y sociales.

Se han investigado los efectos de guanfacina en el tratamiento del TDAH en 5 estudios controlados en niños y adolescentes (de 6 a 17 años), en 3 ensayos controlados a corto plazo en niños y adolescentes de 6 a 17 años, en un estudio controlado a corto plazo en adolescentes de 13 a 17 años y en un ensayo de retirada aleatorizada en niños y adolescentes de 6 a 17 años, de los cuales todos cumplieron los criterios de TDAH del DSM-IV-TR. La mayoría de los pacientes alcanzaron una dosis óptima de entre 0,05 y $0,12 \mathrm{mg} / \mathrm{kg} /$ día.

Intuniv $^{\circledR}$ redujo los síntomas conductuales del TDAH principalmente la hiperactividad, impulsividad y/o corta capacidad de atención y distracción.

Las reacciones adversas más comunes observadas durante el desarrollo clínico fueron: somnolencia, cefalea, fatiga, dolor abdominal y sedación. Las reacciones adversas notificadas más graves incluyeron hipotensión, aumento de peso, bradicardia y síncope. 


\section{INHIBIDOR ALFA 1-PROTEINASA HUMANA $\left(\text { Respreeza }^{\circledR}\right)^{5,12}$}

El principio activo es el inhibidor de la alfa1-proteinasa humana, una alfa1-antitripsina, derivada de sangre humana. La administración del inhibidor alfa1-proteinasa llegará a los pulmones donde restablecerá el nivel de alfa1-antitripsina en pacientes con deficiencia. La alfa1-antitripsina tiene la función de inactivar algunas sustancias como la elastasa, que normalmente las produce el organismo, inhibiendo su efecto. Se espera que esta acción disminuya el empeoramiento del enfisema pulmonar.

Está indicado como tratamiento de mantenimiento para reducir la progresión del enfisema en adultos con deficiencia grave documentada del inhibidor alfa1-proteinasa (por ejemplo: genotipos PiZZ, PiZ (nulo), Pi (nulo, nulo), PiSZ). Los pacientes deben estar bajo tratamiento óptimo farmacológico y no farmacológico y mostrar evidencia de enfermedad pulmonar progresiva (por ejemplo: disminución del volumen espiratorio forzado predictivo en 1 segundo (VEF1), disminución de la capacidad para deambular o incremento en el número de exacerbaciones), evaluado por un médico con experiencia en el tratamiento de sustitución del inhibidor de alfa1-proteinasa humana.

Respreeza $^{\circledR}$ ha demostrado reducir la tasa anual de disminución de densidad pulmonar comparado con placebo a 2 años, medida por escáner, con una reducción del 34\%. Este efecto se ha evaluado en un estudio aleatorio, doble ciego, controlado con placebo y multicéntrico.

Las reacciones adversas más comunes observadas durante el desarrollo clínico fueron hipersensibilidad o reacciones alérgicas observadas durante el tratamiento. En los casos más graves, las reacciones alérgicas pueden progresar a reacciones anafilácticas graves, aun cuando el paciente no ha mostrado hipersensibilidad en administraciones previas.

\section{ISAVUCONAZOL (Cresemba $\left.{ }^{\circledR}\right)^{1,13}$}

El principio activo es isavuconazol, un agente antifúngico triazólico.

Su efecto fungicida se basa en la inhibición de la síntesis del ergosterol, un componente clave de la membrana celular fúngica, mediante la inhibición del enzima 14 alfa-lanosterol desmetilasa.

Está indicado para el tratamiento de adultos con:

- aspergilosis invasiva

- mucormicosis en pacientes en los que la administración de anfotericina $\mathrm{B}$ es inadecuada

El programa de fase 3 con isavuconazol incluye tres estudios, SECURE, VITAL y ACTIVA. El estudio SECURE fue un estudio aleatorizado, doble ciego y evaluó la seguridad y eficacia de isavuconazol una vez al día frente a voriconazol dos veces al día en el tratamiento primario de la enfermedad fúngica invasiva causada por especies de Aspergillus y otros hongos filamentosos en 516 pacientes. Isavuconazol demostró no inferioridad frente a voriconazol en la mortalidad por todas las causas a la semana 42 de tratamiento, que fue del $18.6 \%$ vs $20.2 \%$ con voriconazol. La aprobación para mucormicosis se basó en el estudio VITAL de un solo brazo, en el que 37 pacientes tratados con isavuconazol se compararon con la progresión de la enfermedad natural asociado con la mucormicosis sin tratar. La mortalidad por todas las causas de los pacientes con isavuconazol fue de $38 \%$ mientras que la mortalidad habitual para esta enfermedad es del 50\%. El estudio ACTIVE evalúa la seguridad y eficacia de isavuconazol por vía IV y por vía oral frente a caspofungina IV seguido de voriconazol oral en el tratamiento de la candidemia y otras infecciones invasivas causadas por levaduras del género Candida, y aún no está finalizado.

Las reacciones adversas más comunes observadas durante el desarrollo clínico fueron: elevación de las enzimas hepáticas, náuseas, vómitos, disnea, dolor abdominal, diarrea, reacciones en el sitio de inyección, dolor de cabeza, hipopotasemia y erupción.

Cresemba $^{\circledR}$ fue designado como medicamento huérfano el 4 de julio de 2014 para el tratamiento de la aspergilosis invasiva, y el 4 de junio de 2014 para el tratamiento de la mucormicosis.

\section{PANOBINOSTAT $\left(\text { Farydak }^{\circledR}\right)^{5,14}$}

El principio activo es panobinostat, un inhibidor de histona deacetilasa. Al inhibir la actividad enzimática de la histona deacetilasa se incrementa la acetilación de las histonas, lo que lleva a una activación transcripcional.

Está indicado para el tratamiento del mieloma múltiple refractario y/ o en recaída en combinación con bortezomib y dexametasona en pacientes adultos que han recibido al menos dos tratamientos previos incluyendo bortezomib y un agente inmunomodulador.

Panobinostat, en combinación con bortezomib y dexametasona, ha mostrado su capacidad para retrasar la progresión de la enfermedad, que fue de 12,0 meses en el grupo de panobinostat frente a 8,1 meses en el grupo placebo. La supervivencia global (SG) fue la variable secundaria clave. El número definitivo de acontecimientos de SG no se ha alcanzado todavía. En el segundo análisis intermedio de SG después de haberse observado 359 (86,5\%) de los 415 acontecimientos establecidos para el análisis final de SG, la mediana de SG fue de 38,24 meses en el brazo panobinostat + bortezomib + dexametasona y 35,38 meses en el brazo placebo + bortezomib + dexametasona.

Las reacciones adversas más comunes observadas durante el desarrollo clínico fueron diarrea, fatiga, náuseas, vómitos, trombocitopenia, anemia, neutropenia y linfopenia.

Debe ser prescrito por un médico con experiencia en el tratamiento del cáncer.

Farydak $^{\circledR}$ fue designado como medicamento huérfano el 8 de noviembre de 2012.

\section{PLASMODIUM FALCIPARUM Y VACUNA CONTRA LA HEPATITIS B RECOMBINANTE ADYUVADA (Mosquirix $\left.^{\circledR}\right)^{1,15}$}

El principio activo de Mosquirix ${ }^{\circledR}$ es la proteína circumsporozoito de Plasmodium falciparum fusionada con un antígeno de superficie del virus de la hepatitis B, que se presenta en forma de partículas no infecciosas del virus obtenidas en células de levadura (Saccharomyces cerevisiae) mediante tecnología de ADN recombinante.

Está indicado para la inmunización activa frente a la malaria causada por Plasmodium falciparum y frente a la hepatitis B, en niños desde las 6 semanas hasta los 17 meses de edad. Mosquirix ${ }^{\circledR}$ debe utilizarse de acuerdo con las recomendaciones oficiales tenien- 


\section{Evaluación positiva de medicamentos: junio, julio y agosto 2015}

do en cuenta la epidemiología de la malaria causada por $P$. falciparum en las diferentes áreas geográficas.

Se trata de una vacuna preeritrocítica que limita la capacidad de $P$. falciparum de infectar, crecer y multiplicarse en el hígado mediante la obtención de la inmunidad humoral y celular de la proteína circumsporozoito. Además, Mosquirix ${ }^{\circledR}$ induce una protección frente al virus de la hepatitis $\mathrm{B}$ mediante la obtención de respuestas de inmunidad humoral.

En los ensayos realizados ha mostrado capacidad para proporcionar protección frente a la malaria en niños de entre 6 semanas hasta los 17 meses de edad. En los niños de 6 a 12 semanas, la eficacia de la vacuna frente al primer o único episodio clínico de malaria tras 12 meses de seguimiento fue del $31 \%$, mientras que en los niños de 5 a 17 meses fue del $56 \%$. Durante un período de seguimiento de más de 36 meses, la eficacia de la vacuna frente a todos los episodios de malaria fue del $27 \%$ en los niños de 6 a 12 semanas, y el $46 \%$ en los de 5 a 17 meses.

Las reacciones adversas más comunes observadas durante el desarrollo clínico fueron fiebre, reacciones en el lugar de inyección e irritabilidad.

Este medicamento está destinado exclusivamente para su comercialización fuera de la Unión Europea.

\section{SEBELIPASA ALFA $\left(\text { Kanuma }^{\circledR}\right)^{5,16}$}

El principio activo es sebelipasa alfa, una lipasa ácida lisosomal humana recombinante que ha mostrado capacidad para reemplazar la actividad de la enzima deficitaria, con la consiguiente reducción del contenido de grasa hepática, y de los niveles plasmáticos de transaminasas, colesterol LDL, colesterol No -HDL y triglicéridos. Además, hay un beneficio significativo en términos de supervivencia (67\%) en niños mayores de 12 meses con enfermedad de Wolman.

Está indicada para el tratamiento de sustitución enzimática a largo plazo en pacientes de todas las edades con deficiencia de lipasa ácida lisosomal (LAL). La deficiencia de LAL es una enfermedad rara asociada a una morbimortalidad significativa, que afecta a personas desde la infancia hasta la edad adulta. La deficiencia de LAL que se presenta en lactantes es una urgencia médica con una progresión rápida de la enfermedad durante un periodo de semanas, que suele ser mortal durante los 6 primeros meses de vida.

Las reacciones adversas más graves, experimentadas en un 3\% de los pacientes en los ensayos clínicos, fueron signos y síntomas consistentes con una reacción anafiláctica. Los signos y síntomas incluyeron malestar en el pecho, inyección conjuntival, disnea, erupción generalizada y prurito, hiperemia, edema palpebral leve, rinorrea, dificultad respiratoria grave, taquicardia, taquipnea y urticaria.

Debe ser prescrito por médicos con experiencia en el tratamiento del déficit de lipasa ácida liposomal u otros desórdenes metabólicos o insuficiencia hepática crónica.

Kanuma $^{\circledR}$ fue designado como medicamento huérfano el 17 de diciembre de 2010 .

\section{SONIDEGIB $\left(\text { Odomzo }^{\circledR}\right)^{5,17}$}

El principio activo es sonidegib, un agente antineoplásico que inhibe la vía de señalización Hedgehog, un regulador clave en el desarrollo y la morfogénesis de los mamíferos, la cual está unida a la patogénesis de varios cánceres incluyendo carcinoma basocelular (CBC).

Está indicado para el tratamiento de pacientes adultos con $\mathrm{CBC}$ localmente avanzado y no candidatos para cirugía o radioterapia.

Sonidegib ha mostrado reducir las lesiones del CBC localmente avanzado con una tasa de respuesta del $54 \%$ y una supervivivencia libre de progresión de aproximadamente 22 meses.

Las reacciones adversas más comunes observadas durante el desarrollo clínico fueron calambres musculares, dolor muscular, pérdida de cabello, alteración o pérdida del gusto, náuseas, diarrea, incremento en sangre de creatinfosfoquinasa, pérdida de peso, y fatiga.

Se implementarán un plan de prevención de embarazos y un plan de farmacovigilancia como parte de la autorización de comercialización.

Debe ser prescrito por o bajo la supervisión de médicos con experiencia en el manejo de la indicación aprobada.

\section{SUSOCTOCOG ALFA $\left(\text { Obizur }^{\circledR}\right)^{1,18}$}

El principio activo es susoctocog alfa, un factor VIII recombinante porcino. Detiene el sangrado mediante la formación de un complejo con el factor de coagulación IX activado y acelerando la conversión del factor X en factor X activado. Este convierte la protrombina en trombina y éste convierte el fibrinógeno en fibrina de forma que se pueda formar un coágulo.

En los ensayos clínicos ha mostrado su capacidad para detener o reducir el sangrado con la consiguiente mejoría clínica.

Está indicado en adultos para el tratamiento de episodios hemorrágicos en pacientes con hemofilia adquirida causada por anticuerpos de los factores de coagulación VIII. En base a los datos de eficacia y seguridad aportados se recomienda una autorización bajo circunstancias excepcionales. La autorización en circunstancias excepcionales está sujeta a ciertas obligaciones específicas, y se revisa de forma anual. Esto sucede cuando el solicitante no es capaz de proporcionar datos exhaustivos sobre eficacia y seguridad del medicamento, debido a la baja frecuencia de la enfermedad para la que está destinada, conocimientos científicos limitados en el área concerniente o consideraciones éticas relacionadas con la recogida de datos.

La reacción adversa más común observada durante el desarrollo clínico fue el desarrollo de anticuerpos inhibidores contra el factor VIII porcino.

El tratamiento debe iniciarse bajo la supervisión de un médico con experiencia en el trata-miento de la hemofilia.

Obizur $^{\circledR}$ fue designado como medicamento huérfano el 20 de septiembre de 2010 .

\section{BIBLIOGRAFIA}

1. Agencia Española de Medicamentos y Productos Sanitarios. Informe mensual sobre medicamentos de Uso Humano y Productos Sanitario. Julio 2015. Disponible en URL: http://www.aemps.gob.es/informa/boletinMensual/2015/julio/docs/ boletin-mensual_julio-2015.pdf

2. Committee for Medicinal Products for Human Use (CHMP) EMA CHMP/392428/2015 23 July 2015. Disponible en URL: http://www.ema.europa eu/docs/en_GB/document_library/Summary_of_opinion_-_Initial_authorisation/human/003882/WC500190416.pdf 


\section{P. Prats Olivan, et al.}

3. Kereiakes DJ, Robinson JG, Cannon CP, Lorenzato C, Pordy R, Chaudhari U, Colhoun HM. Efficacy and safety of the proprotein convertase subtilisin/kexin type 9 inhibitor alirocumab among high cardiovascular risk patients on maximally tolerated statin therapy: The ODYSSEY COMBO I study. Am Heart J. 2015 Jun; 169(6):906-915.

4. Robinson JG, Farnier M, Krempf M, Bergeron J, Luc G, Averna M, Stroes ES, Langslet G, Raal FJ, El Shahawy M, Koren MJ, Lepor NE, Lorenzato C, Pordy R, Chaudhari U, Kastelein JJ; ODYSSEY LONG TERM Investigators. Efficacy and safety of alirocumab in reducing lipids and cardiovascular events. N Engl J Med. 2015 Apr 16; 372(16):1489-99.

5. Agencia Española de Medicamentos y Productos Sanitarios. Informe mensual sobre medicamentos de Uso Humano y Productos Sanitario. Junio 2015. Disponible en URL: http://www.aemps.gob.es/informa/boletinMensual/2015/junio/ docs/boletin-mensual junio-2015.pdf

6. Committee for Medicinal Products for Human Use (CHMP) EMA/ CHMP/383497/2015 25 June 2015. Disponible en URL: http://www.ema.europa.eu/docs/en_GB/document_library/Summary_of_opinion_-_Initial_authorisation/human/003794/WC500188778.pdf

7. Committee for Medicinal Products for Human Use (CHMP) EMA/ CHMP/448694/2015 23 July 2015. Disponible en URL: http://www.ema.europa. eu/docs/en_GB/document_library/Summary_of_opinion_-_Initial_authorisation/human/003772/WC500190407.pdf

8. Wagenlehner FM, Umeh O, Steenbergen J, Yuan G, Darouiche RO. Ceftolozane-tazobactam compared with levofloxacin in the treatment of complicated urinary-tract infections, including pyelonephritis: a randomised, double-blind, phase 3 trial (ASPECT-cUTI). Lancet. 2015 May 16; 385(9981):1949-56

9. Committee for Medicinal Products for Human Use (CHMP) EMA CHMP/453873/2015 25 June 2015. Disponible en URL: http://www.ema.europa.eu/docs/en_GB/document_library/Summary_of_opinion_-_Initial_authorisation/human/003776/WC500190451.pdf

10. Committee for Medicinal Products for Human Use (CHMP) EMA/ CHMP/535898/2015 30 July 2015. Disponible en URL: http://www.ema.europa.eu/docs/en_GB/document_library/EPAR__Public_assessment_report/human/003776/WC500194873.pdf
11. Committee for Medicinal Products for Human Use (CHMP) EMA CHMP/450088/2015 23 July 2015. Disponible en URL: http://www.ema.europa eu/docs/en_GB/document_library/Summary_of_opinion_-_Initial_authorisation/human/003759/WC500190423.pdf

12. Committee for Medicinal Products for Human Use (CHMP) EMA/ CHMP/76738/2015 25 June 2015. Disponible en URL: http://www.ema.europa eu/docs/en_GB/document_library/Summary_of_opinion_-_Initial_authorisation/human/002739/WC500188773.pdf

13. Committee for Medicinal Products for Human Use (CHMP) EMA CHMP/470149/2015 23 July 2015. Disponible en URL: http://www.ema.europa eu/docs/en_GB/document_library/Summary_of_opinion_-_Initial_authorisation/human/002734/WC500190471.pdf

14. Committee for Medicinal Products for Human Use (CHMP) EMA/ CHMP/304387/2015 25 June 2015. Disponible en URL: http://www.ema.europa.eu/docs/en_GB/document_library/Summary_of_opinion_-_Initial_authorisation/human/003725/WC500188792.pdf

15. Committee for Medicinal Products for Human Use (CHMP) EMA/ CHMP/464758/2015 23 July 2015. Disponible en URL: http://www.ema.europa. eu/docs/en_GB/document_library/Other/2015/07/WC500190452.pdf

16. Committee for Medicinal Products for Human Use (CHMP) EMA/ CHMP/400811/2015 25 June 2015. Disponible en URL: http://www.ema.europa.eu/docs/en_GB/document library/Summary of opinion - Initial authorisation/human/004004/WC500188768.pdf

17. Committee for Medicinal Products for Human Use (CHMP) EMA CHMP/304387/2015 25 June 2015. Disponible en URL: http://www.ema.europa.eu/docs/en_GB/document_library/Summary_of_opinion_-_Initial_authorisation/human/002839/WC500188762.pdf

18. Committee for Medicinal Products for Human Use (CHMP) EMA/ CHMP/304387/2015 25 June 2015. Disponible en URL: http://www.ema.europa eu/docs/en_GB/document_library/Summary_of_opinion_-_ _Initial_authorisation/human/002792/WC500190472.pdf 